\title{
Improvement of the Mechanical and Biomedical Properties of Implants via the Production of Nanocomposite Based on Nanostructured Titanium Matrix and Bioactive Nanocoating
}

\author{
E.G. Zemtsova, A.Yu. Arbenin, R.Z. Valiev and V.M. Smirnov
}

\begin{abstract}
The work describes the complex approach for production of the biomaterial that is able for the accelerated osteosynthesis (i.e., rate of the implants engraftment into the bone tissue). The combined approach is based on bulk nanostructuring of titanium matrix and surface nanostructuring based on bioactive porous coating. This approach leads to enhancement of both mechanical and biomedical properties of implants. We created bioactive coating on nano-Ti substrates using modeling the oxide layer structure on nano and micro level. The developed bioactive surfaces with two-level surfaces allow to control surface relief both on micro and nano level with high precision $(1 \mathrm{~nm})$. These surfaces do not lead to the degradation of the mechanical properties of nanotitanium. Biomedical studies showed that the composite coating demonstrates high surface adhesion properties for the osteoblasts MC3T3-E1 cell line. Along with adhesion, also initial differentiation of osteoblasts is observed. This indicates the ability of the surface to the accelerated osteosynthesis. The developed nano-Ti-based composite nanomaterial with bioactive nanocoating can be used for the production of the new generation implants for dentistry, reconstructive surgery and orthopedics.
\end{abstract}

E.G. Zemtsova $(\bowtie) \cdot$ A.Yu. Arbenin · R.Z. Valiev · V.M. Smirnov

Saint Petersburg State University, Saint Petersburg, Russia

e-mail: ezimtsova@yandex.ru

A.Yu. Arbenin

e-mail: aua47@yandex.ru

R.Z. Valiev

e-mail: rzvaliev@gmail.com

V.M. Smirnov

e-mail: vms11@yandex.ru

(C) The Author(s) 2018

K.V. Anisimov et al. (eds.), Proceedings of the Scientific-Practical Conference

"Research and Development - 2016", https://doi.org/10.1007/978-3-319-62870-7_49 
Keywords Composite biomaterial $\cdot$ Nanostructured titanium $\cdot$ Bioactive nanocoating - Chemical modification of surface $\cdot$ Surface relief Titanooragnic coatings - Calcium-Phosphate structures - Method of molecular layering (ml-ald) • Sol-gel synthesis • Osteointegration • Titanium implants

To the moment, traditionally used metallic biomaterials mostly reached their maximum of tensile strength. For application in medicine, these biomaterials must satisfy the set of requirements. On the one hand, they should be bioactive and biocompatible. On the other hand, such materials should demonstrate high mechanical strength especially under cyclic loads. This is an important property to ensure durability of medical products.

The increase of human life expectancy as well as the progress of modern surgery require the development of the metallic biomaterials (implants) that demonstrate accelerated engraftment (improved bioactive properties). According to the clinical statistics, the problem of rejection is relevant even for the latest models of implants. There is also the problem of the huge healing period, which is not comfortable for the patients.

Most of bioactive coating studies in Russia and worldwide are dedicated either to change of surface relief or to variation of the surface layer composition. However, acceptable coating from the point of view of the osteointegration acceleration is still not found. It was shown earlier that elemental composition of the implant surface and the titanium surface relief play important role in the osteoblasts (young bone cells) formation and in the increase of their biocompatibility [7, p. 2727]. Qualitative and quantitative indicators of osteointegration directly depend on Ti-implant surface topography and chemical composition [8, p. $87 ; 10$, p. 5]. In this regard, an important direction of the medical materials science is to develop surface modification methods, which will increase osteocompatibility between implants and tissues.

The goal of this work is the development of the scientific basics of nanotitanium-based composite material with bioactive nanocoating. The combined approach is based on bulk nanostructuring of titanium matrix by means of severe plastic deformation and surface nanostructuring based on bioactive porous coating. This approach leads to increase of mechanical and biomedical properties of implants. We reached the accelerated osteosynthesis for enhancement of the rate of implants engraftment. Also the life of the product is increased and whole-life implantation is provided. Improved mechanical properties of the material give miniaturized implant construction. This effect decreases injury surface and accelerates osteosynthesis.

The developed bioactive surfaces with two-level surfaces allow to control surface relief both on micro and nano level with high precision $(1 \mathrm{~nm})$. Moreover, these surfaces do not lead to the degradation of the mechanical properties of nanotitanium.

For application in medicine, the titanium-based biomaterials must satisfy the set of requirements. On the one hand, they need to be bioactive and biocompatible. On 
the other hand, such medical materials must possess considerable mechanical strength, especially under action of the cyclic loads. This is important to ensure the durability of medical products.

In this work, we applied severe plastic deformation (SPD) approach that represents powerful mechanical action on the material. By means of ECAP-Conform, the structure is formed with the average grain size of $100 \mathrm{~nm}$, and high level of the mechanical characteristics (tensile strength $(\sigma \mathrm{B})-1240 \mathrm{MPa})$. It is established that high level of the mechanical characteristics without loss of plasticity is provided by the specific properties of the formed grain boundaries and their high densities in the nano-Ti structure. Application of nanostructured Ti allows to miniaturize implants construction keeping unchanged its exploitation properties.

The joint replacement is actively developed within the modern orthopedics. One of the novel important materials providing fast implants engraftment is bioactive coatings. A lot of scientific groups work in this direction: there are researches on the possibility of introducing of various organic compounds into the surface layer [8, p. 87], coating implants with polymers - both biogenic [9, p. 1025], or synthetic [11, p. 4135]. However, as it is mentioned in some works [2, p. 217; 3, p. 889], along with the chemical composition, another key factor that determines bioactivity is the coating relief. Cytological and histological analysis indicates the efficiency of the coatings with two-level surface relief organization on micro- and nanolevel [4, p.].

In this work, the complex approach is suggested for the generation of bioactive coating on nanotitanium matrices. This attained by means of modelling of the oxide layer structure and composition, as well as coating thickness on the nano scale.

As a matrix, nanotitanium samples were used that were produced from Grade 4 titanium according to [12, p. 28]. Surface topography was examined by scanning probe microscopy (Solver P47 Pro in the tapping mode on air) and scanning tunnel microscopy (Zeiss Supra 40VP). The research was performed in the Interdisciplinary Research Center for Nanotechnology of Saint Petersburg State University (SPbSU). According to atomic force microscopy (AFM), the surface of initial Ti substrate after mechanical and chemical treatment is characterized by very low roughness level. The average height difference was $\sim 3 \mathrm{~nm}$.

We used ALD method in order to produce the nanocoating with controlled structure relief. ALD is based on the surface chemical reactions that take place on the selected substrate $[13$, p. 590]. The main advantage of this method is the ability to produce nanostructures (thin films) with high precision (Angstroms) with controlled surface geometry and roughness.

It is also worth to note high adhesion of nanocoatings to the titanium substrate. This effect is reached due to consecutive cycling chemisorption of low molecular reagents from the gas phase. Surfaces with predicted roughness were produced by controlling the number of treatment cycles, which led to increasing length of Ti-organic nanostructures (from 5 to 20 cycles) [14, p. 374].

It was shown that after 20 cycles of ALD treatment of nanotitanium, initial surface is fully covered by Ti-organic nanostructures. The distance between nanostructures varies from 75 to $120 \mathrm{~nm}$; the average size of nanostructures is $120 \mathrm{~nm}$. Roughness height lies between 75 and $200 \mathrm{~nm}$ (Fig. 1). 
In vitro studies of the samples were used to examine structure characteristics on the adhesion, proliferation and differentiation rate of osteoblasts MC3T3-E1. Osteocompatibility of the samples was compared with one for titanium having the natural $\mathrm{TiO}_{2}$ layer on the surface. The cell state (adhesion and spreading of the cells on the sample surface) was done using SEM.

The sample of nano-Ti with Ti-organic nanostructures demonstrates the pronounced cells monolayer with high adhesion properties of the surface for osteoblasts MC3T3-E1 cell line (Fig. 2). Almost twofold growth of proliferation and viability of cells MC3T3-E1 is observed compared to the control samples without coating. Along with adhesion, also initial differentiation of osteoblasts cells is observed. This effect indicates the ability of the surface to the accelerated osteosynthesis.

So, almost twofold increase of osteogenic differentiation markers production in vitro comparing to the control, allows to predict that time of engraftment will be decreased. This, in turn, leads to reducing duration of hospitalization and

(a)

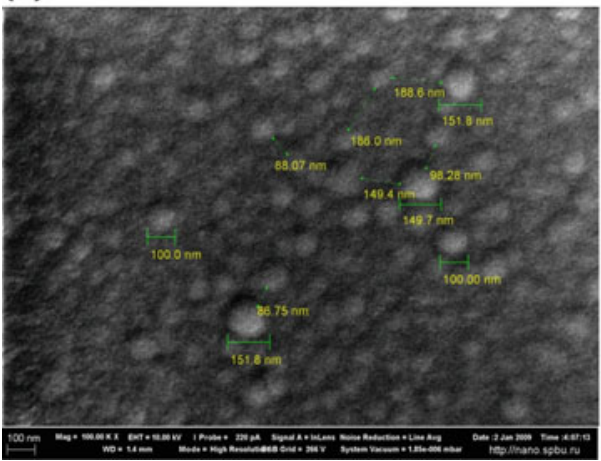

(b)

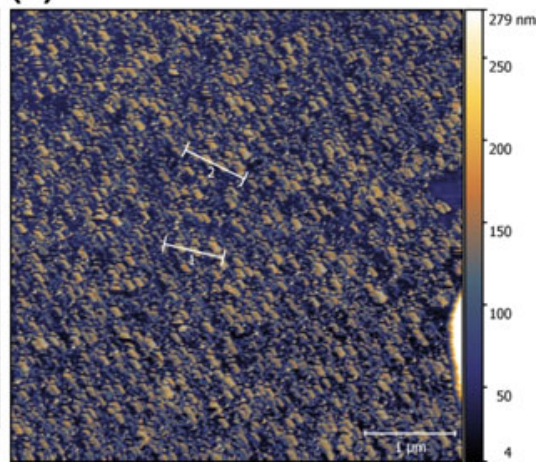

Fig. 1 Analysis of nanotitanium surface with coated Ti-organic nanostructures: a microtopography of the sample surface, $\mathbf{b}$ AFM reconstruction of the sample surface

(a)

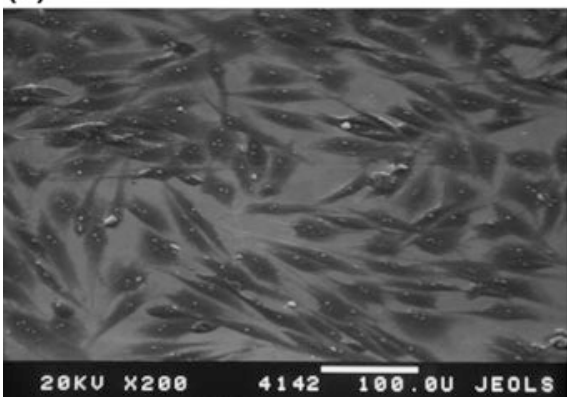

(b)

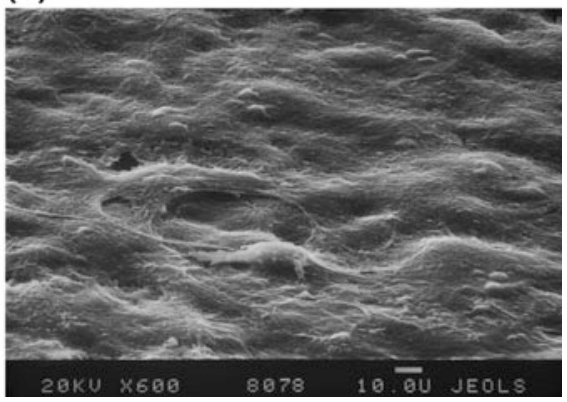

Fig. 2 Electronic microphotograph of the surface after osteoblasts incubation: a initial Ti, b surface with coated Ti-organic nanostructures 
rehabilitation of patients. Our experimental data suggest that cells viability and their growth depend not only on the chemical composition of the coating but also on surface relief (i.e., its roughness).

Taking into account the requirement that the metallic implants (e.g., titanium dental implants) should have strongly rough surface on the nanolevel, we can conclude that ALD method is really prospective for the production of the bioactive nanocoatings.

Also we developed the complex approach of the modelling of oxide layer structure on the nano and micro level. $\mathrm{TiO}_{2}$-based nanocoating with two-level relief organization are produced by sol-gel method in the dip coating mode. For this nanocoating, roughness is observed both on nano and micro level. Two-level hierarchy is realized by applying shock drying on hot plate at $150-500{ }^{\circ} \mathrm{C}[1$, p. 2453]. Micron-level structure is raised due to erosion of the continuous film. Nano-level structure is caused by granular relief of the film (pore size of 5-20 nm). Oxide layer thickness is varied by means of cyclization of dip coating procedure. As a result, the samples were produced after 1-5 cycles of the nano-Ti substrate treatment. The coatings with thickness between 70 and $200 \mathrm{~nm}$ were obtained. Spectral ellipsometry data indicate the linear dependence of the $\mathrm{TiO}_{2}$ film thickness on the number of layers. The developed approach can lead to large variation of film textures on the nano-Ti substrate: from nearly smooth film until the dense cracks net on the micro level. For the first time, we produced composite coating on the nano- $\mathrm{Ti}$ surface $\left(\mathrm{TiO}_{2} /\right.$ calcium-phosphate composite), that looks like $\mathrm{TiO}_{2}$ islands with inclusions of calcium-phosphate structures.

The coating with the thickness of $150 \mathrm{~nm}$ with the developed net of microcracks and pore size of 5-20 nm (Fig. 3) demonstrates high adhesion properties for the studied cells line MC3T3-E1. Also, along with adhesion, we observed continuous cells layer with osteocyte channels.

The induction period for the osteoblasts differentiation is diminished due to the two-level relief hierarchy (Fig. 4). This indicates the ability of the surface to the accelerated osteosynthesis.

(a)

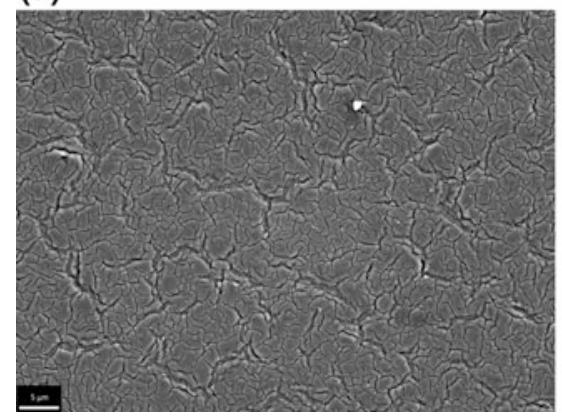

(b)

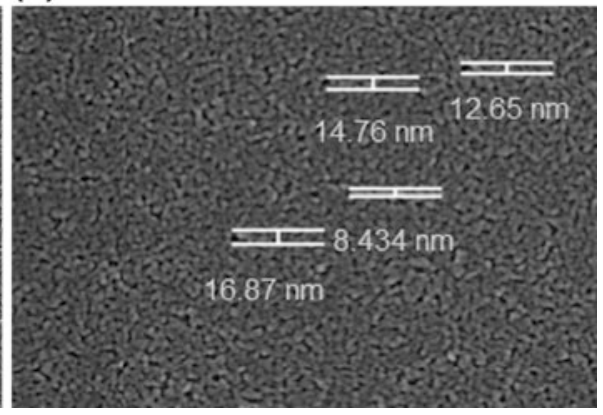

Fig. 3 Microphotographs of the surface of $\mathrm{TiO}_{2}$ layer produced by sol-gel method in the dip coating mode (thickness $150 \mathrm{~nm}$ ): a microstructure, b nanostructure 
(a)

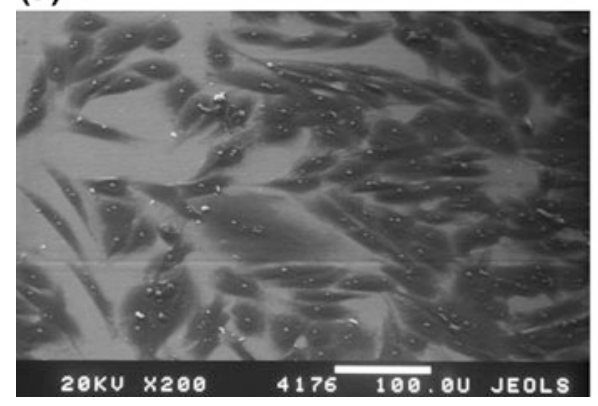

(b)

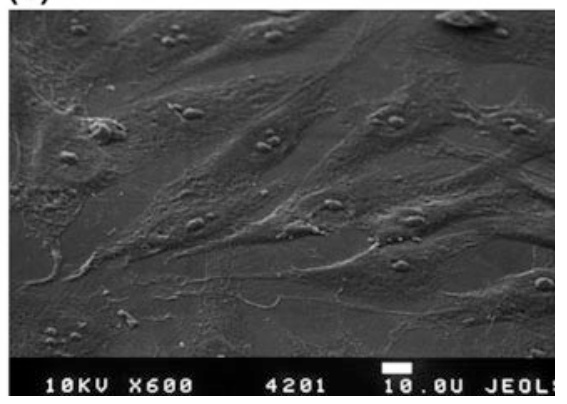

Fig. 4 Electronic microphotograph of the surface after osteoblasts incubation: $\mathbf{a}$ initial $\mathrm{Ti}, \mathbf{b} \mathrm{TiO}_{2}$ layer produced by sol-gel method in the dip coating mode (thickness is $150 \mathrm{~nm}$ )

According to some references [6, p. 387; 7, p. 2727] the presence of surface roughness (both on micro and nanolevel) is the key factor for osteoblasts adsorption on the implant surface.

Our data suggest the absence of cytotoxicity of the studied coatings. This is consistent with the earlier data [5, p. 397].

The developed technology of the production of nano-Ti based composite nanomaterial with bioactive nanocoating as well as this material itself, can be used for the production of the new generation implants for dentistry, reconstructive surgery and orthopedics. While variating chemical composition of the bioactive coating, the material can be used as the implant that substitutes mineralized tissues of various skeletal parts.

\section{Competitive Advantages of Our Approach}

- Possibility to produce nanostructured coating with the high precision $(1 \mathrm{~nm})$;

- possibility for directed regulation of geometry and roughness of the surface in the nano scale;

- coating with two-level surface relief that is controlled both on micro and nanolevel;

- absence of cytotoxicity of suggested coatings;

- the coatings do not lead to diminishing mechanical properties of nanostructured titanium, tensile strength is retained $(1240 \mathrm{MPa})$;

- miniaturization of constructions, enhancement of mechanical properties of the implants;

- improvement of functional properties due to bulk and surface nanostructuring allows twofold accelerating of the implants engraftment into human body. The service life of the implant grows manifold. 
- high adhesion properties of the implants surface for the studied cell line, ability to the accelerated osteosynthesis (rate of implants engraftment in the human body).

\section{Conclusions}

We developed the method of production of nanostructured titanium matrix (nanotitanium) with enhanced strength without plasticity loss. The improved mechanical properties of nanostructured titanium can assist in the future in the miniaturization of implant construction.

Nanotitanium with titan-organic nanostructures on the surface was developed. Biomedical studies showed that the composite coating with surface roughness between 75 and $200 \mathrm{~nm}$ with mean pore size of $120 \mathrm{~nm}$ demonstrates high surface adhesion properties for the osteoblasts MC3T3-E1 cell line. It is shown that together with adhesion, initial differentiation of osteoblasts is observed. This indicates the ability of the surface to the accelerated osteosynthesis.

Based on the analysis of the mechanical properties, we established that synthesized coatings do not lead to the degradation of mechanical characteristics of nanotitanium. We developed the approach of the synthesis of the nanotitanium samples with porous nanostructured $\mathrm{TiO}_{2}$ films on the surface with inclusion of calcium-phosphate structures. The modification of the surfaces by porous mesostructured $\mathrm{TiO}_{2}$ films up to $180 \mathrm{~nm}$ thick, with pore size from $5 \mathrm{~nm}$ to $20 \mathrm{~nm}$ and microrelief is optimal not only for the stimulation of MC3T3-E1 cells proliferation but also for their differentiation in the osteogenic direction. The study of the adhesion activity of MC3T3-E1 cells demonstrates that osteoblasts undergo adhesion on the surface of all the studied samples for all the cultivation times ( $24 \mathrm{~h}, 7$ or $14 \mathrm{~d})$. They have morphology characteristic for this cell line. The adhesion ability of the osteoblasts MC3T3-E1 is more than $85 \%$ for $72 \mathrm{~h}$.

This work is a scientific study that contributes to the development of a new generation of clinically significant metallic biomaterials (bioactive and biocompatible implants). These materials play important role in the human life enhancement and prolongation. The materials are used in the medicine (dentistry, orthopedics, traumatology) in order to keep life and normal functioning of the organism.

Acknowledgements Research are carried out with the financial support of the state represented by the Ministry of Education and Science of the Russian Federation. Contract no. 14.604.21.0084 30. Jun 2014. Unique project Identifier: RFMEFI 60414X0084. 


\section{References}

1. Arbenin, A.Yu., Zemtsova, E.G., Valiev, R.Z., Smirnov, V.M.: Russ. J. Gen. Chem. 84(12), 2453-2454 (2014)

2. Bucci-Sabattini, V., Cassinelli, C., Coelho, P.G., Minnici, A., Trani, A., Dohan Ehrenfest, D.M.: Oral Maxillofac. 109, 217-224 (2010)

3. Buser, D., Schenk, R.K., Steinemann, S., Fiorellini, J.P., Fox, C.H., Stich, H.: J. Biomed. Mater. Res. 25, 889-902 (1991)

4. Dalby, M.J., McCloy, D., Robertson, M., Wilkinson, C.D.W., Oreffo, R.O.C.: Biomaterials 27, 1306-1315 (2006)

5. Geetha, M.: Ti based biomaterials, the ultimate choice for orthopaedic implants. A review. Prog. Mater. Sci. 54, 397-425 (2009)

6. Hench, Larry L., Jones, Julian R. (eds.): Biomaterials, Artificial Organs and Tissue Engineering, p. 304. Woodhead publishing limited, Cambridge, England (2005)

7. Lilja, M., Genvad, A., Astrand, M., Strømme, M., Enqvist, H.: Influence of microstructure and chemical composition of sputter deposited $\mathrm{TiO}_{2}$ thin films on in vitro bioactivity. J. Mater. Sci. Mater. Med. 22, 2727-2734 (2011)

8. Chen, Q., Thouas, G.A.: Metallic implant biomaterials. Mater. Sci. Eng. R. 87, 1-57 (2015)

9. Rammelt, S., Schulze, E., Bernhardt, R., Hanisch, U., Scharnwebe, Dr., Worch, H., Zwipp, H., Biewener, A.: J. Orthop. Res. 22, 1025-1034 (2004)

10. Singh, P.P.: Drug alluding dental implant Patent № US 0208148-A1. (2012)

11. Singh Harris, L.G., Tosatti, S., Wieland, Textor, M., Richards, R.G.: Biomaterials 25, 4135 4148 (2004)

12. Valiev Ruslan, Z., Zhilyaev Alexander, P., Langdon Terence, G.: Bulk Nanostructured Materials: Fundamentals and Applications. Wiley, Hoboken, 439 p, (2014)

13. Smirnov, V.M.: Russ. J. Gen. Chem. 72, 590-607 (2002)

14. Zemtsova, E.G., Morozov, P.E., Smirnov, V.M.: Regulation of surface topography of nanostructured titanium using the method of ML-ALD to create bioactive nanocoatings. Mater. Phys. Mech. 24, 374-381 (2015)

Open Access This chapter is licensed under the terms of the Creative Commons Attribution 4.0 International License (http://creativecommons.org/licenses/by/4.0/), which permits use, sharing, adaptation, distribution and reproduction in any medium or format, as long as you give appropriate credit to the original author(s) and the source, provide a link to the Creative Commons license and indicate if changes were made.

The images or other third party material in this chapter are included in the chapter's Creative Commons license, unless indicated otherwise in a credit line to the material. If material is not included in the chapter's Creative Commons license and your intended use is not permitted by statutory regulation or exceeds the permitted use, you will need to obtain permission directly from the copyright holder. 